\section{Etiquetado de Alimentos en Chile: comentarios a la carta sobre la Ley 30021 de Perú}

\section{Food labeling in Chile: Comments on the letter regarding Law 30021 in Peru}

\author{
Marcela Reyes Jedlicki ${ }^{1}$ \\ 'Doctora en Nutrición y Alimentos. Instituto de Nutrición y \\ Tecnología de los Alimentos, Universidad de Chile. Santiago \\ de Chile, Chile. $\square$ iD
}

doi: $10.18294 /$ sc. 2018.1948

Comentarios sobre: Arista Fernández H, Mundaca Rojas KG, Sosa Flores J, Torres Anaya V. Ley 30021 de Promoción de Alimentación Saludable para Niños, Niñas y Adolescentes. Salud Colectiva. 2018;14(3):639-640. doi: $10.18294 /$ sc.2018.1679.

En la carta titulada "Ley 30021 de Promoción de Alimentación Saludable para Niños, Niñas y Adolescentes" dirigida al editor se aprecia la frustración de un grupo de médicos y de estudiantes de medicina de Perú con relación a la iniciativa de instaurar un etiquetado frontal de los alimentos que favorezca las decisiones saludables en alimentación. La malnutrición en todas sus formas es un problema en continuo aumento en todo el mundo, pero de forma particularmente rápida en América Latina ${ }^{(1,2)}$. Esta se manifiesta en el aumento del sobrepeso y la obesidad, tanto en adultos como en niños, incluso en familias en las que coexiste la desnutrición ${ }^{(3)}$. Esta situación, junto con los llamados que hicieron las diferentes organizaciones internacionales de salud, incluida la Organización Panamericana de la Salud (OPS), ha hecho que muchos países de la región estén tomando diferentes medidas para mejorar la dieta de sus poblaciones.

Hay muchos factores que moldean la alimentación de las poblaciones, por lo que esta puede ser mejorada de diferentes maneras. Dado el poco efecto que han tenido diversas iniciativas de educación en nutrición y salud para la toma de decisiones más saludable, las iniciativas que se están discutiendo actualmente tienen el foco en modificar los factores del ambiente que influyen en las decisiones alimentarias, en lugar de enfocarse en los factores individuales. La idea es favorecer las decisiones saludables a través de la modificación de la disponibilidad de alimentos, sus precios, las estrategias promocionales que usan, las normas socioculturales, entre otros factores del ambiente alimentario(4)

Uno de estos factores ambientales es el etiquetado de los alimentos. En muchos países, es obligatorio informar en los envases el contenido de los principales nutrientes y de ingredientes de los alimentos envasados previo a su venta. Las características de este etiquetado están reguladas y pueden variar de país a país. Los estudios que se han realizado sugieren que este tipo de etiquetado es usado y entendido por una minoría de la población, lo que sucede de forma transversal en diferentes países, aún en aquellos con mejores niveles de educación.

Hace ya varias décadas surgieron las primeras iniciativas de ofrecer un etiquetado que comunique de manera más simple la información sobre la calidad nutricional de los alimentos. A lo largo de los años, se han propuesto múltiples alternativas, que varían en el nivel de detalle de la información, en su diseño, o según se enfoquen en las características positivas o negativas del alimento, entre otras características. La gran mayoría de estas experiencias ha sido implementada de forma optativa, por lo que es difícil estimar el impacto. En junio de 2016, se implementó en Chile la Ley № 20.606 sobre la Composición Nutricional de los Alimentos y su Publicidad, que señala (entre otras cosas) la obligatoriedad del uso de un símbolo octogonal negro, con letras blancas, indicando "alto en energía", "alto en azúcares", "alto en sodio", o "alto en grasas saturadas" cuando los alimentos envasados superan límites específicos de estos nutrientes. Por lo tanto, hace más de dos años que en Chile los 
alimentos que están envasados previo a su venta pueden tener hasta cuatro signos pare, indicando su condición de altos en nutrientes críticos. Cabe señalar, que contrariamente a lo que dice la carta, este etiquetado frontal fue desarrollado por un grupo de académicos de la Universidad de Chile, a solicitud de las autoridades de salud, y no fue una propuesta de la industria de alimentos.

La medida es aún muy reciente como para esperar cambios relevantes en el estado nutricional de la población, pero los estudios que se han hecho evaluando la implementación $^{(5)}$ sugieren que la gente valora la medida, la usa para saber qué tan saludable son los alimentos y consume menos (en cantidad o frecuencia) de los alimentos con más signos pare. Si bien esto no sucede en toda la población, la medida parece ser adecuada dado que, según los diferentes reportes, aproximadamente el $50 \%$ de los entrevistados señalan estar usando las etiquetas de acuerdo con lo esperado por las autoridades de salud, lo que desde una perspectiva de salud pública es un éxito.

Algunos meses antes de que Perú adoptara el sistema de etiquetado octogonal, los autores de la carta expresaban su preocupación por el posible cambio del signo pare por el semáforo, como etiquetado frontal para los alimentos que se vendan en Perú. El semáforo es un sistema implementado de forma optativa en el Reino Unido hace más de una década y, en 2014, de forma obligatoria en Ecuador. Para su aplicación se fijan rangos de: 1) bajo o saludable (color verde), 2) intermedio (color naranja), y 3) alto o poco saludable (color rojo), para cada uno de los nutrientes de interés. De esta forma, un alimento puede tener hasta tres colores diferentes para grasas, azúcares y sodio. Si bien los estudios muestran que esta simbología representa un avance con relación a la información nutricional tradicional ${ }^{(6,7)}$ y es mejor entendida, estudios recientes ${ }^{(8,9,10)}$ realizados por investigadores uruguayos y brasileños, que comparan los signos pare con el semáforo, muestran claramente cómo los primeros son más efectivos en términos de ser identificados en los envases, de comunicar la información de una manera comprensible para el consumidor y de mejorar la selección de alimentos (haciéndola más saludable).

Si bien se puede pensar que una etiqueta de alimentos que entrega más información es más eficiente en comunicar lo que quiere comunicar (qué tan saludable o no saludable es dicho alimento), esto no es así para el caso de las decisiones alimentarias. Esto puede deberse a una mezcla de hechos, por ejemplo, la gran complejidad del tema nutricional, que hace que la información deba ser entregada de la manera más sencilla posible. Por otra parte, debemos considerar que muchas de nuestras decisiones no son racionales. Hay decisiones que tenemos que hacer muy frecuentemente, como es el caso de la alimentación (varias veces al día). Esas decisiones, en general, se hacen con poca reflexión, y son diferentes a otras que se toman pocas veces en la vida, como la compra de un auto, o con quien casarse. De esta manera, si bien las luces del semáforo ayudan a entender qué tan saludable es un alimento, a la hora de decisiones cortas y frecuentes, es mucho más probable que -si se hace algún esfuerzo cognitivo- este sea contar el número de signos pare negros, en lugar de tratar de entender si 2 verdes +1 naranja +1 rojo es más o menos saludable que 1 verde +3 naranjas.

En junio de 2018, a través del Decreto Supremo No. 012-2018-SA ${ }^{(11)}$, el gobierno de Perú puso en vigencia el Manual de Advertencias Publicitarias, por lo que no queda más que celebrar el resultado del proceso peruano.

\section{REFERENCIAS BIBLIOGRÁFICAS}

1. Popkin BM, Reardon T. Obesity and the food system transformation in Latin America. Obesity Reviews. 2018;19:1028-1064. doi: 10.1111/obr.12694.

2. NCD Risk Factor Collaboration. Worldwide trends in body-mass index, underweight, overweight, and obesity from 1975 to 2016: a pooled analysis of 2416 populationbased measurement studies in 128.9 million children, adolescents, and adults. Lancet. 2017;390:2627-2642. doi: 10.1016/ S0140-6736(17)32129-3.

3. Rivera JA, Pedraza LS, Martorell R, Gil A. Introduction to the double burden of undernutrition and excess 
weight in Latin America. American Journal of Clinical Nutrition. 2014;100(6):S1613-S1616. doi: 10.3945/ ajcn.114.084806.

4. Swinburn B, Vandevijvere S, Kraak V, Sacks G, Snowdon W, Hawkes C, Barquera S, Friel S, Kelly B, Kumanyika S, L'Abbé M, Lee A, Lobstein T, Ma J, Macmullan J, Mohan S, Monteiro C, Neal B, Rayner M, Sanders D, Walker C, INFORMAS. Monitoring and benchmarking government policies and actions to improve the healthiness of food environments: a proposed Government Healthy Food Environment Policy Index. Obesity Reviews. 2013;14(Suppl 1):24-37. doi: 10.1111/obr.12073.

5. Chile, Ministerio de Salud. Reglamento de la Ley de Etiquetado de Alimentos evaluación [Internet]. 2017 [citado 10 feb 2018]. Disponible en: https://tinyurl.com/ y9gd4sd8.

6. Graham DJ, Heidrick C, Hodgin K. Nutrition label viewing during a food-selection task: Front-of-package labels vs nutrition facts labels. Journal of the Academy of Nutrition and Dietetics. 2015;115(10):1636-1646. doi: 10.1016/j.jand.2015.02.019.

7. Becker MW, Sundar RP, Bello N, Alzahabi R, Weatherspoon L, Bix L. Assessing attentional prioritization of front-of-pack nutrition labels using change detection. Applied Ergonomics. 2016;54:90-99. doi: 10.1016/j. apergo.2015.11.014

8. Arrúa A, Curutchet MR, Rey N, Barreto P, Golovchenko N, Sellanes A, Velazco G, Winokur M, Giménez A, Ares G. Impact of front-of-pack nutrition information and label design on children's choice of two snack foods: Comparison of warnings and the traffic-light system. Appetite. 2017;116:139-146. doi: 10.1016/j. appet.2017.04.012

9. Zoellner JM, Hedrick VE, You W, Chen Y, Davy BM, Porter KJ, Bailey A, Lane H, Alexander R, Estabrooks PA. Effects of a behavioral and health literacy intervention to reduce sugar-sweetened beverages: a randomizedcontrolled trial. International Journal of Behavioral Nutrition and Physical Activity. 2016;13:38. doi: 10.1186/ s12966-016-0362-1.

10. Khandpur N, Sato PM, Mais LA, Martins APB, Spinillo CG, Garcia MT, Rojas CFU, Jaime PC. Are front-ofpackage warning labels more effective at communicating nutrition information than traffic-light labels?: A randomized controlled experiment in a Brazilian sample. Nutrients. 2018;10(6):688. doi: 10.3390/nu10060688.

11. Diario Oficial El Peruano. Aprueban Manual de Advertencias Publicitarias en el marco de lo establecido en la Ley No 30021, Ley de promoción de la alimentación saludable para niños, niñas y adolescentes, y su Reglamento aprobado por Decreto Supremo No 017-2017-SA [Internet]. 16 jun 2018 [citado 10 ago 2018]. Disponible en: https://tinyurl.com/ya7mwzv3.

\section{FORMA DE CITAR}

Reyes Jedlicki M. Etiquetado de Alimentos en Chile: comentarios a la carta sobre la Ley 30021 de Perú. Salud Colectiva. 2018;14(3):641-643. doi: 10.18294/ sc. 2018.1948

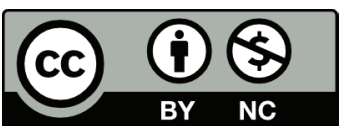

Este obra está bajo una licencia de Creative Commons Reconocimiento-NoComercial 4.0 Internacional. Reconocimiento - Permite copiar, distribuir y comunicar públicamente la obra. A cambio, se debe reconocer y citar al autor original. No Comercial - Esta obra no puede ser utilizada con finalidades comerciales, a menos que se obtenga el permiso. 